\title{
Evaluation of variability in Some Local Genotypes of Maize (Zea mays L) under rain fed at Damazin Research Station,Blue Nile State
}

\author{
Mohmed Osmsn A.A. ${ }^{1}$, Osman A.M. ${ }^{2}$, Abu Assar A.H. ${ }^{3}$, Dagash.Y.M.I ${ }^{1, *}$ \\ ${ }^{1}$ Department of Agronomy, College of Agric. Studies, SUST, Khartoum, Sudan \\ ${ }^{2}$ Blue Nile University.Damazin, Sudan \\ ${ }^{3}$ Wad Medani Research Corporation. Sudan \\ *Corresponding Author: dagash501@sustech.edu
}

Copyright (C) 2014 Horizon Research Publishing All rights reserved.

\begin{abstract}
Estimation of the genetic variability among 18 local genotypes of Maize (Zea mays L) was recorded. In a genotype $\mathrm{X}$ tester fashion, 16 genotypes were crossed with low testers. All the $32 \mathrm{~F}_{1}$ genotype and their 18 parents were sown for two seasons (2007/08 and 2008/09), in summer (Autum). The results in season 2007/08 indicated highly significant differences for Number of leaves / plant while significant for the--- character--- day's to $50 \%$ tasselling, stem diameter, leaf area, $\mathrm{Cob}$ bareness \% Cob diameter, $\mathrm{Cob}$ weight, yield/ plant and yield ( $t / h a)$. On the other hands in season 2008/09, a highly significant differences were shown for three characters (day's to $50 \%$ tasselling, Cob diameter and yield $\mathrm{t} / \mathrm{ha}$ ), while significant for six characters (stem diameter, Number of leaves/Plant, leaf area, Cob bareness\%, Cob weight and yield per plant).
\end{abstract}

Keywords Maize, Hybrid, Yield and Damazin Area

\section{Introduction}

Maize (Zea mays L) ranks as one of the worlds' three most important cereal crop. It is cultivated in wider range of environments than wheat and rice because of its greater adaptability (Koutsika and Sotiriou, 1999). Currently, its global production area is about 140 million hectares, of which approximately 96 million hectares in the developing countries. Although $68 \%$ of the world maize area is in developing countries, $46 \%$ of the world's maize production of 602 million tons (FAO, 2003) is produced in other area. Low average yields in the tropics are responsible for the wide gap between the global shore of area and of production (Heisey and Edmeades, 1999 and Pingali and pandey, 2000).
Drought and low $\mathrm{N}_{2}$ stresses are factors most frequently limiting maize production in the tropics (Edmeades et al., 1997; Banziger et al., 1999b and Vasal et al., 1997; 1999).

The objective of the study is to evaluate 50 local maize genotypes from Blue Nile State for yield and yield components.

\section{Materials and Methods}

This trail was carried out in summer (Autum of 2007 and 2008) for two seasons at Damazin Research Station Farm. Blue Nile State $\left(11^{\circ} 57.460 \mathrm{~N}\right.$, lat. and $34^{\circ}-13.547$ E. long). The genetic materials used in this study (Table 1) consisted of 50 local maize, using a Randomized Complete Block Design (RCBD) with four replications. Each block (replicate) was divided into plots, to which the hybrids and their parents were assigned randomly. The plot sizes were four rows of 4 meters length $70 \mathrm{Cm}$ spacing between rows and $20 \mathrm{Cm}$ between hills. Planting rate was made as three seeds / hole. These were then thinned to one plant / hole after two weeks from sowing. Sowing practiced was on $12^{\text {th }}$ of July in season 2007 and $14^{\text {th }}$ of July 2008 . Weeding was carried out when needed. One row at each side of the plot was treated as a boarder row to avoid the inter row competitions. Ten randomly selected plants from the middle of the two rows of each plot were used to record the data on the following: Days to $50 \%$ tasselling, stem diameter, Number of leaves| plant, leaf area, Cob bareness\%, Cob diameter, Cob weight, yield| plant and yield $/(\mathrm{t} / \mathrm{ha})$. The collected data were analyzed based on individual and combined analysis of variance. The procedure described by Gomez and Gomez (1984) were used to estimate the individual and combined analysis of variance. 
Table 1. 50 local maize (genetic materials) used in the study

\begin{tabular}{|c|c|c|}
\hline Genotype code & Pedigree & Type \\
\hline 1 & $\mathrm{M}_{45}$ & Tester \\
\hline 2 & $\mathrm{H}_{1}$ & Tester \\
\hline 3 & $\mathrm{~B}_{1}$ & Local (lines) \\
\hline 4 & $\mathrm{~B}_{2}$ & Local (lines) \\
\hline 5 & $\mathrm{~B}_{3}$ & Local (lines) \\
\hline 6 & $\mathrm{~B}_{4}$ & Local (lines) \\
\hline 7 & $\mathrm{~B}_{5}$ & Local (lines) \\
\hline 8 & $\mathrm{D}_{1}$ & Local (lines) \\
\hline 9 & $\mathrm{D}_{2}$ & Local ( lines) \\
\hline 10 & $\mathrm{D}_{3}$ & Local (lines) \\
\hline 11 & $\mathrm{D}_{4}$ & Local (lines) \\
\hline 12 & $\mathrm{D}_{5}$ & Local (lines) \\
\hline 13 & $\mathrm{D}_{6}$ & Local (lines) \\
\hline 14 & $\mathrm{R}_{1}$ & Local (lines) \\
\hline 15 & $\mathrm{R}_{2}$ & Local (lines) \\
\hline 16 & $\mathrm{R}_{3}$ & Local (lines) \\
\hline 17 & $\mathrm{R}_{4}$ & Local (lines) \\
\hline 18 & $\mathrm{R}_{5}$ & Local (lines) \\
\hline \multicolumn{3}{|c|}{$\mathrm{F}_{1}$ Hybrids } \\
\hline 19 & $\mathrm{~B}_{1} \times \mathrm{M}_{45}$ & Local \\
\hline 20 & $\mathrm{~B}_{2} \times \mathrm{M}_{45}$ & Local \\
\hline 21 & $\mathrm{~B}_{3} \times \mathrm{M}_{45}$ & Local \\
\hline 22 & $\mathrm{~B}_{4} \times \mathrm{M}_{45}$ & Local \\
\hline 23 & $\mathrm{~B}_{5} \times \mathrm{M}_{45}$ & Local \\
\hline 24 & $\mathrm{~B}_{1} \times \mathrm{H}_{1}$ & Local \\
\hline 25 & $\mathrm{~B}_{2} \times \mathrm{H}_{1}$ & Local \\
\hline 26 & $\mathrm{~B}_{3} \times \mathrm{H}_{1}$ & Local \\
\hline 27 & $\mathrm{~B}_{4} \times \mathrm{H}_{1}$ & Local \\
\hline 28 & $\mathrm{~B}_{5} \times \mathrm{H}_{1}$ & Local \\
\hline 29 & $\mathrm{D} 1 \times \mathrm{M} 45$ & Local \\
\hline 30 & $\mathrm{D}_{2} \times \mathrm{M}_{45}$ & Local \\
\hline 31 & $\mathrm{D}_{3} \times \mathrm{M}_{45}$ & Local \\
\hline 32 & $\mathrm{D}_{4} \times \mathrm{M}_{45}$ & Local \\
\hline 33 & $\mathrm{D}_{5} \times \mathrm{M}_{45}$ & Local \\
\hline 34 & $\mathrm{D}_{6} \times \mathrm{M}_{45}$ & Local \\
\hline 35 & $\mathrm{D}_{1} \times \mathrm{H}_{1}$ & Local \\
\hline 36 & $\mathrm{D}_{2} \times \mathrm{H}_{1}$ & Local \\
\hline
\end{tabular}




\begin{tabular}{|c|c|c|}
\hline 37 & $\mathrm{D}_{3} \times \mathrm{H}_{1}$ & Local \\
\hline 38 & $\mathrm{D}_{4} \times \mathrm{H}_{1}$ & Local \\
\hline 39 & $\mathrm{D}_{5} \times \mathrm{H}_{1}$ & Local \\
\hline 40 & $\mathrm{D}_{6} \times \mathrm{H}_{1}$ & Local \\
\hline 41 & $\mathrm{R}_{1} \times \mathrm{M}_{45}$ & Local \\
\hline 42 & $\mathrm{R}_{2} \times \mathrm{M}_{45}$ & Local \\
\hline 43 & $\mathrm{R}_{3} \times \mathrm{M}_{45}$ & Local \\
\hline 44 & $\mathrm{R}_{4} \times \mathrm{M}_{45}$ & Local \\
\hline 45 & $\mathrm{R}_{5} \times \mathrm{M}_{45}$ & Local \\
\hline 46 & $\mathrm{R}_{1} \times \mathrm{H}_{1}$ & Local \\
\hline 47 & $\mathrm{R}_{2} \times \mathrm{H}_{1}$ & Local \\
\hline 48 & $\mathrm{R}_{3} \times \mathrm{H}_{1}$ & Local \\
\hline 49 & $\mathrm{R}_{4} \times \mathrm{H}_{1}$ & Local \\
\hline 50 & $\mathrm{R}_{5} \times \mathrm{H}_{1}$ & Local \\
\hline
\end{tabular}

\section{Results \& Discussion}

\subsection{Days to $50 \%$ Tasselling}

There was highly significant differences $(\mathrm{p} \leq .01)$ among the evaluated maize genotypes, in days to $50 \%$ tasselling---- in second season while significant in first season (Table 2). The genotype $X$ tester analysis of variance (Table 3 ) showed highly significant differences for crosses in second and-non-significant for genotypes and tester, and location in first season -----. The combined analysis of variance Table (4) showed highly significant differences for genotype and non- significant for season X genotypes ( $\mathrm{SXG}$ ). The earliest genotype was $\mathrm{D}_{4}$ (54.4 day) during the first season and the Latest--- were $\mathrm{M}_{45} \mathrm{X}$ $\mathrm{D}_{3}$ (61.1day), while in the second season, the latest one were $\mathrm{M}_{45} \mathrm{X}_{3}\left(61.1\right.$ day), and in the 2 nd season the earliest were $\mathrm{H}_{\mathrm{I}} \mathrm{X}$ $\mathrm{R}_{3}$ (54.8 day) and $\mathrm{H}_{1} \mathrm{X} \mathrm{R}_{2}$ (60.4 day) is the latest one. The overall mean days to $50 \%$ tasselling was $57.8 \%$ in both seasons. During the first season, the earliest genotype was $\mathrm{R}_{5}$ (54.7 day) during the first season and $\mathrm{H}_{1} \mathrm{X} \mathrm{R} 1$ (60.4 days) were the latest. In the second season, $\mathrm{D}_{5}\left(54.9\right.$ day) were the earliest and $\mathrm{H}_{1} \mathrm{X} \mathrm{R}_{2}$ (61.8day) was the latest one. The overall mean days to $50 \%$ tasselling were 57.2 and 58.0 days in the first and the second season respectively. The coefficient of variations was $2.121 \%$ in the first season and 3.93 in the second season.

Table 2. Mean square from analysis of varies for different characters in fifty maize genotypes evaluated during season 2007/2008 at Blue Nile State.

\begin{tabular}{|c|c|c|}
\hline \multirow{2}{*}{ Character } & \multicolumn{2}{|c|}{ Menotype } \\
\cline { 2 - 3 } & \multicolumn{2}{|c|}{ Mean squares } \\
\hline Day to 50\% tasselling & $22.1^{*}$ & $18.3^{* *}$ \\
\hline Stem diameter $(\mathrm{Cm})$ & $20.7^{*}$ & $18.4^{*}$ \\
\hline Leaf/ plant & $3.1^{* *}$ & $2.7^{*}$ \\
\hline Leaf/ area(Cm $\left.{ }^{2}\right)$ & $16086.1^{*}$ & $121023.3^{*}$ \\
\hline Cob parents & $0.9^{*}$ & $0.8^{*}$ \\
\hline Cob diameter(Cm) & $0.3^{*}$ & $0.2^{* *}$ \\
\hline Cob weight(g) & $927.6^{*}$ & $811.4^{*}$ \\
\hline yield/ plant & $2162.1^{*}$ & $2045.3^{*}$ \\
\hline yield ton/ ha & $6.1^{*}$ & $5.8^{* *}$ \\
\hline
\end{tabular}

$*=$ Significant

** = Highly significant

$\mathrm{NS}=$ Non significant 
Table 3. Mean squares from the analysis of variance for some morphological characters in ferity maize genotype evaluated Damazin season 2007

\begin{tabular}{|c|c|c|c|c|c|}
\hline Characters & $\begin{array}{c}\text { Crosses } \\
\text { d.f }=31\end{array}$ & $\begin{array}{c}\text { Lines } \\
\text { d.f }=15\end{array}$ & $\begin{array}{c}\text { Tester } \\
\text { d.f }=1\end{array}$ & $\begin{array}{c}\text { Lines } x \text { tester } \\
\text { d.f }=15\end{array}$ & $\begin{array}{c}\text { Error } \\
\text { d.f }=147\end{array}$ \\
\hline Day to $50 \%$ tasselling & $11.57^{* *}$ & $14.04 \mathrm{~ns}$ & $2.20 \mathrm{~ns}$ & $9.72 \mathrm{~ns}$ & 6.10 \\
\hline Stem diameter & $0.43^{* *}$ & $0.49 \mathrm{~ns}$ & $0.02 \mathrm{~ns}$ & $0.40^{* *}$ & 0.07 \\
\hline Leaf/ plant & $5.22^{* *}$ & $6.02 \mathrm{~ns}$ & $1.40 \mathrm{~ns}$ & $4.67^{* *}$ & 0.63 \\
\hline Leaf/ area & $15798.90^{* *}$ & $12330.88 \mathrm{~ns}$ & $78.75 \mathrm{~ns}$ & $20314.92^{* *}$ & 1628.35 \\
\hline Cob parenss & $1.64^{* *}$ & $2.88^{* *}$ & $0.43 \mathrm{~ns}$ & $0.47^{* *}$ & 0.15 \\
\hline Cob diameter & $0.52^{* *}$ & $0.52 \mathrm{~ns}$ & $4.69 \mathrm{~ns}$ & $0.25^{*}$ & 0.12 \\
\hline Cob weight & $561.45^{* *}$ & $553.83 \mathrm{~ns}$ & $2349.55^{*}$ & $449.87^{* *}$ & 21.12 \\
\hline yield/ plant & $108.15^{* *}$ & $144.42 \mathrm{~ns}$ & $5.61 \mathrm{~ns}$ & $78.72^{* *}$ & 8.29 \\
\hline yield ton/ ha & $2.59^{* *}$ & $2.27 \mathrm{~ns}$ & $3.03 \mathrm{~ns}$ & $2.88^{* *}$ & 0.17 \\
\hline
\end{tabular}

$*=$ Significant

** = Highly significant

NS $=$ Non significant

Table 4. Mean squares from the analysis of variance for some morphological characters in 50 maize genotype evaluated Damazin season 2008

\begin{tabular}{|c|c|c|c|c|c|}
\hline Characters & $\begin{array}{l}\text { Crosses } \\
\text { d.f }=31\end{array}$ & $\begin{array}{l}\text { Lines } \\
\text { d.f }=15\end{array}$ & $\begin{array}{l}\text { Tester } \\
\text { d.f }=1\end{array}$ & $\begin{array}{c}\text { Lines } x \text { tester } \\
\text { d.f }=15\end{array}$ & $\begin{array}{c}\text { Error } \\
\text { d.f }=147\end{array}$ \\
\hline Day to $50 \%$ tasselling & $9.92 *$ & $13.36 \mathrm{~ns}$ & $3.51 \mathrm{~ns}$ & $6.90 \mathrm{~ns}$ & 6.03 \\
\hline Stem diameter & $0.39^{* *}$ & $0.32 \mathrm{~ns}$ & $0.20 \mathrm{~ns}$ & $0.47^{* *}$ & 0.08 \\
\hline Leaf/ plant & $5.48^{* *}$ & $5.05 \mathrm{~ns}$ & $0.01 \mathrm{~ns}$ & $6.28^{\star \star}$ & 1.10 \\
\hline Leaf/ area & $11158.35^{\star *}$ & $12169.95 \mathrm{~ns}$ & $23620.51 \mathrm{~ns}$ & $9315.94^{* *}$ & 1581.27 \\
\hline Cob parenss & $1.64^{* *}$ & $2.88^{* *}$ & $0.43 n s$ & $0.47^{* *}$ & 0.15 \\
\hline Cob diameter & $0.52^{\star *}$ & $0.52 \mathrm{~ns}$ & $4.69^{* *}$ & $0.25^{*}$ & 0.12 \\
\hline Cob weight & $561.45^{* *}$ & $553.83 \mathrm{~ns}$ & $2349.55^{\star}$ & $449.87^{* *}$ & 21.12 \\
\hline yield/ plant & $108.15^{\star \star}$ & $144.42 \mathrm{~ns}$ & $5.61 \mathrm{~ns}$ & $78.72^{\star *}$ & 8.29 \\
\hline yield ton/ ha & $3.33^{* *}$ & $1.46 \mathrm{~ns}$ & $58.19^{* *}$ & $1.54^{* *}$ & 0.24 \\
\hline
\end{tabular}

$$
\begin{aligned}
& *=\text { Significant } \\
& * *=\text { Highly significant } \\
& \text { NS= Non significant }
\end{aligned}
$$

Table 5. Mean square from the combined analysis of variance for some morphological character in 50 maize genotypes evaluated Damazin for two seasons 2007/2008

\begin{tabular}{|l|l|l|l|l|l|l|l|l|l|}
\hline $\begin{array}{l}\text { Source of } \\
\text { variation }\end{array}$ & $\begin{array}{l}\text { Day to 50\% } \\
\text { tasselling }\end{array}$ & $\begin{array}{l}\text { Stem } \\
\text { diamete } \\
\text { ( }(\mathrm{cm})\end{array}$ & $\begin{array}{l}\text { Leaf/ } \\
\text { plant }\end{array}$ & $\begin{array}{l}\text { Leaf/ } \\
\text { area } \\
\left(\mathrm{cm}^{2}\right)\end{array}$ & $\begin{array}{l}\text { Cob } \\
\text { bareness } \\
(\%)\end{array}$ & $\begin{array}{l}\text { Cob } \\
\text { diameter } \\
(\mathrm{cm})\end{array}$ & $\begin{array}{l}\text { Cob } \\
\text { weight } \\
(\mathrm{g})\end{array}$ & $\begin{array}{l}\text { yield/ } \\
\text { plant }(\mathrm{g})\end{array}$ & $\begin{array}{l}\text { yield ton/ } \\
\text { ha }\end{array}$ \\
\hline Rep/yrs d.fb & 4.29 & - & - & 4968.43 & 0.04 & 0.20 & 32.87 & 28.16 & 0.24 \\
\hline Season (s)dsl & $0.67^{\mathrm{ns}}$ & - & - & $\begin{array}{l}30923.22 \\
* *\end{array}$ & $0.00^{\mathrm{ns}}$ & $0.00^{\mathrm{ns}}$ & $0.00^{\mathrm{ns}}$ & $0.00^{\mathrm{ns}}$ & $4.62^{* *}$ \\
\hline $\begin{array}{l}\text { Genotype(G) } \\
\text { f44 }\end{array}$ & $20.82^{* *}$ & - & - & 26366.11 & $2.84^{* *}$ & $0.97^{* *}$ & $1362.16^{*}$ & $378.77^{* *}$ & $3.85^{* *}$ \\
\hline Sx G.df49 & $1.30^{\mathrm{ns}}$ & - & - & $2358.76^{*}$ & $0.00^{\mathrm{ns}}$ & $0.00^{\mathrm{ns}}$ & $0.00^{\mathrm{ns}}$ & $0.00^{\mathrm{ns}}$ & $4.53^{* *}$ \\
\hline $\begin{array}{l}\text { Pooled 279 } \\
\text { error ds }\end{array}$ & 6.07 & - & - & 1604.81 & 0.15 & 0.12 & 21.12 & 8.29 & 0.20 \\
\hline
\end{tabular}

*= Significant

** = Highly significant

$\mathrm{NS}=$ Non significant 


\subsection{Stem Diameter}

The genotype $\mathrm{X}$ tester analysis of variance recorded highly significant differences for crosses in both seasons and locations, but for genotypes and tester non- significant differences was obtained in both seasons. The combined analysis of variance Table (4) showed significant differences, for seasons, genotypes, and non-signification for genotypes $\mathrm{X}$ seasons, The largest stem diameter were $\mathrm{M}_{45} \mathrm{X} \mathrm{B}_{1}(3.1 \mathrm{Cm})$ in the first season and the smallest genotype were $\mathrm{R}_{4}(1.6$ $\mathrm{Cm})$ in the second season, the $\mathrm{M}_{45} \mathrm{X} \mathrm{B}_{1}(3.0 \mathrm{Cm})$ and the lowest one were detected by the genotype $\mathrm{M}_{45} \times \mathrm{B}_{5}(1.7$ $\mathrm{Cm}$ ), with the overall mean of stem diameter of 2.1 and 2.2 $\mathrm{Cm}$ in the first and second season respectively, and the coefficient of variation of $12.766 \%$ in the first season and $13.01 \%$ in the second season.

\subsection{Number of Leave Per Plant}

Individual analysis of variance revealed significant differences, and indicated that the significant difference among the genotype in first season and highly significant differences in second season (Table 2). The genotype $\mathrm{X}$ tester analysis of variance (Table 3) showed highly significant differences for the crosses in both seasons and locations. Non significant differences were showed for genotype and testers in both seasons and location, while highly significant differences were recorded for genotype $\mathrm{X}$ teeter in both seasons and location. The combined analysis of variance showed highly significant for season, genotype and season genotype in location (Table 4). The highest number of leaves /plant was reported by genotype $\mathrm{B}_{2}(15.2)$ as shown in Table (6) and the lowest leaves per plant by genotype $\mathrm{MX} \mathrm{B}_{2}$ (11.0) in the first season, while in the second season the highest leaves per plant was reported by genotype $\mathrm{B}_{2}$ (14.9) and the lowest one was given by genotype $\mathrm{M}_{45} \mathrm{X} \mathrm{D}_{4}$ (11.2), with the overall mean number of leaves per plant as 13.0 in the first season and 13.1 in the second one with the coefficient of variation of $6.09 \%$ and $7.99 \%$ in the first and second season, respectively.

Table 6. Means of the different character in 50 maize genotype evaluated Blue Nile in season 2007 at Damazin

\begin{tabular}{|c|c|c|c|c|c|c|c|c|c|}
\hline Genotype & $\begin{array}{c}\text { Days to } 50 \% \\
\text { Tasseling }\end{array}$ & $\begin{array}{c}\text { Stem } \\
\text { diameter } \\
(\mathrm{CM}) \\
\end{array}$ & $\begin{array}{c}\text { Number of } \\
\text { leaves } \\
\text { /plant }\end{array}$ & $\begin{array}{l}\text { leaf area } \\
(\mathrm{cm})\end{array}$ & $\begin{array}{c}\text { Cob } \\
\text { Barness }\end{array}$ & $\begin{array}{c}\text { Cob } \\
\text { diameter } \\
(\mathrm{cm}) \\
\end{array}$ & $\begin{array}{c}\text { Cob } \\
\text { weight } \\
(\mathrm{cm}) \\
\end{array}$ & $\begin{array}{l}\text { yield per } \\
\text { plant(g) }\end{array}$ & yield $\mathrm{t} / \mathrm{h}$ \\
\hline M45 & 57.0 & 2.0 & 12.7 & 374.1 & 1.9 & 4.2 & 116.1 & 98.4 & 4.1 \\
\hline $\mathrm{H} 1$ & 57.0 & 1.9 & 11.5 & 392.6 & 1.7 & 3.8 & 112.7 & 106.0 & 4.8 \\
\hline B1 & 55.6 & 2.3 & 13.5 & 408.7 & 1.5 & 3.8 & 120.0 & 84.8 & 4.1 \\
\hline B2 & 61.4 & 2.2 & 15.2 & 302.4 & 1.4 & 4.6 & 107.8 & 84.3 & 4.0 \\
\hline B3 & 56.7 & 2.2 & 12.7 & 269.4 & 1.9 & 3.5 & 95.6 & 99.0 & 3.9 \\
\hline B4 & 57.2 & 1.9 & 13.2 & 275.4 & 1.4 & 3.6 & 125.1 & 86.5 & 4.2 \\
\hline B5 & 56.3 & 2.3 & 12.3 & 307.2 & 1.7 & 4.5 & 113.9 & 81.2 & 4.3 \\
\hline D1 & 56.8 & 2.1 & 13.7 & 422.2 & 1.6 & 4.1 & 118.0 & 76.6 & 4.0 \\
\hline D2 & 58.9 & 2.8 & 14.7 & 377.1 & 1.7 & 4.1 & 119.8 & 83.3 & 4.4 \\
\hline D3 & 56.7 & 1.9 & 13.6 & 292.9 & 1.7 & 4.3 & 112.4 & 85.2 & 4.3 \\
\hline D4 & 54.4 & 1.8 & 12.7 & 405.6 & 2.4 & 3.7 & 94.3 & 81.2 & 4.7 \\
\hline D5 & 55.2 & 2.3 & 12.6 & 355.0 & 2.2 & 4.2 & 98.0 & 77.3 & 4.0 \\
\hline D6 & 57.7 & 2.0 & 11.7 & 407.0 & 1.7 & 3.6 & 95.1 & 76.7 & 5.3 \\
\hline $\mathrm{R} 1$ & 55.9 & 1.9 & 14.8 & 227.0 & 1.5 & 3.8 & 87.9 & 75.3 & 2.0 \\
\hline $\mathrm{R} 2$ & 56.9 & 1.8 & 11.8 & 325.6 & 2.2 & 3.9 & 85.2 & 78.9 & 4.1 \\
\hline R3 & 56.0 & 1.8 & 12.4 & 190.2 & 1.6 & 4.0 & 118.8 & 85.8 & 4.1 \\
\hline $\mathrm{R} 4$ & 57.7 & 1.6 & 13.9 & 368.9 & 2.4 & 3.6 & 111.2 & 88.9 & 4.2 \\
\hline R5 & 59.8 & 2.2 & 15.1 & 338.0 & 2.5 & 3.7 & 100.4 & 83.9 & 4.0 \\
\hline M45XB1 & 60.6 & 3.1 & 11.0 & 302.3 & 2.7 & 4.1 & 93.4 & 83.4 & 3.9 \\
\hline M45XB2 & 56.3 & 2.2 & 14.1 & 479.3 & 1.5 & 3.9 & 95.8 & 81.2 & 3.4 \\
\hline M45XB3 & 59.0 & 2.5 & 13.4 & 351.4 & 2.7 & 4.3 & 118.9 & 74.0 & 3.4 \\
\hline M45XB4 & 58.7 & 1.9 & 13.5 & 294.8 & 3.1 & 3.9 & 105.7 & 83.3 & 3.4 \\
\hline
\end{tabular}




\begin{tabular}{|c|c|c|c|c|c|c|c|c|c|}
\hline M45XB5 & 57.3 & 1.7 & 13.3 & 391.0 & 1.8 & 3.8 & 102.4 & 82.9 & 3.7 \\
\hline M45XD1 & 57.7 & 2.2 & 11.1 & 360.7 & 1.9 & 4.4 & 96.0 & 81.7 & 4.1 \\
\hline M45XD2 & 57.4 & 2.2 & 12.7 & 234.1 & 1.6 & 4.4 & 100.0 & 82.4 & 4.5 \\
\hline M45XD3 & 61.8 & 1.8 & 13.6 & 326.9 & 1.9 & 3.9 & 103.6 & 80.9 & 5.3 \\
\hline M45XD4 & 55.8 & 2.1 & 11.4 & 241.8 & 2.9 & 3.4 & 99.6 & 79.0 & 4.2 \\
\hline M45XD5 & 60.3 & 1.9 & 13.1 & 256.2 & 2.1 & 4.0 & 105.3 & 79.8 & 3.3 \\
\hline M45XD6 & 58.5 & 1.7 & 11.8 & 382.5 & 3.1 & 3.9 & 88.1 & 77.1 & 4.0 \\
\hline M45XR1 & 57.3 & 1.7 & 11.2 & 361.7 & 2.6 & 4.5 & 95.9 & 80.0 & 4.1 \\
\hline M45XR2 & 57.6 & 2.1 & 14.8 & 329.3 & 2.1 & 3.8 & 95.9 & 82.3 & 4.9 \\
\hline M45XR3 & 56.3 & 2.6 & 13.8 & 229.6 & 1.9 & 4.0 & 94.9 & 74.7 & 5.0 \\
\hline M45XR4 & 56.5 & 1.9 & 12.1 & 330.3 & 2.5 & 4.2 & 93.9 & 81.3 & 3.5 \\
\hline M45XR5 & 59.4 & 2.4 & 14.2 & 403.3 & 3.6 & 3.9 & 116.1 & 83.0 & 3.1 \\
\hline H1XB1 & 56.9 & 2.3 & 13.6 & 384.7 & 2.6 & 3.1 & 106.1 & 84.1 & 3.2 \\
\hline H1XB2 & 60.1 & 2.3 & 13.5 & 347.9 & 1.7 & 3.7 & 108.4 & 84.9 & 4.6 \\
\hline H1XB3 & 61.0 & 2.4 & 11.8 & 356.1 & 3.9 & 3.5 & 87.2 & 56.6 & 5.4 \\
\hline H1XB4 & 59.6 & 1.9 & 12.8 & 279.7 & 2.9 & 3.6 & 75.6 & 76.5 & 4.5 \\
\hline H1XB5 & 60.7 & 2.2 & 11.2 & 269.2 & 1.9 & 2.9 & 87.1 & 75.9 & 5.3 \\
\hline H1XD1 & 59.2 & 2.0 & 11.9 & 215.4 & 1.4 & 3.4 & 84.7 & 81.5 & 4.7 \\
\hline H1XD2 & 58.3 & 2.5 & 14.3 & 273.9 & 1.6 & 4.0 & 86.3 & 82.1 & 4.8 \\
\hline H1XD3 & 57.7 & 1.9 & 14.1 & 307.2 & 1.5 & 3.8 & 105.9 & 84.0 & 3.2 \\
\hline H1XD4 & 56.0 & 2.3 & 14.9 & 422.2 & 2.4 & 3.3 & 113.4 & 82.5 & 6.1 \\
\hline H1XD5 & 60.5 & 2.6 & 12.5 & 377.1 & 2.2 & 4.0 & 94.5 & 79.6 & 3.6 \\
\hline H1XD6 & 57.2 & 1.9 & 11.9 & 292.9 & 2.8 & 3.7 & 85.8 & 84.2 & 3.4 \\
\hline H1XR1 & 56.6 & 1.9 & 12.7 & 405.6 & 2.7 & 4.1 & 69.2 & 83.2 & 3.7 \\
\hline H1XR2 & 60.0 & 1.7 & 14.2 & 384.7 & 1.6 & 3.7 & 75.0 & 77.6 & 5.2 \\
\hline H1XR3 & 56.1 & 1.7 & 14.1 & 347.9 & 2.3 & 3.4 & 82.0 & 83.4 & 3.3 \\
\hline H1XR4 & 57.0 & 2.2 & 12.4 & 356.1 & 2.1 & 4.0 & 100.8 & 79.7 & 3.3 \\
\hline H1XR5 & 57.9 & 2.1 & 12.7 & 279.7 & 2.8 & 3.9 & 106.3 & 84.5 & 4.3 \\
\hline $\begin{array}{c}\text { Overall } \\
\text { means }\end{array}$ & 57.8 & 2.1 & 13.0 & 332.3 & 2.1 & 3.9 & 100.1 & 82.0 & 4.1 \\
\hline C.V\% & 4.27 & 12.66 & 6.09 & 12.14 & 17.85 & 9.12 & 4.59 & 3.51 & 9.87 \\
\hline
\end{tabular}

\subsection{Leaf Area $\left(\mathrm{Cm}^{2}\right)$}

The individual analysis of variance for leaf area indicated significant differences among the evaluated genotypes at location in both seasons (Table 2). The mean square for genotype $\mathrm{X}$ tester analysis showed highly significant differences for crosses at location, season. Non- significant for genotypes and testers were detected in both years and location (Table 3). On the other hand, the combined analysis recorded highly significant differences for genotypes $\mathrm{X}$ tester in both seasons and location. The combined analysis of variance showed (table 7) highly significant differences for season, genotypes, and seasons X genotypes. Maximum leaf area were recorded by genotype $\mathrm{M}_{45} \mathrm{X} \mathrm{B}_{2}(479.3 \mathrm{Cm})$, while the minimum one were given by the genotype $\mathrm{R}_{1}(190.2 \mathrm{Cm})$ in the first season, but in the second season, the maximum leaf area were recorded by genotype $\mathrm{M}_{45} \mathrm{X} \mathrm{B}_{2}(453.2 \mathrm{~cm})$ and the minimum one were recorded by genotype $\mathrm{R}_{3}(192.4)$, with the overall mean leaf area of $332.3 \mathrm{Cm}^{2}$ in the first season and $314.7 \mathrm{Cm}^{2}$ in the second one. The coefficient of variation was $12.14 \%$ in the first season and $12.64 \%$ in the second season. 
Table 7. Means of the different character in 50 maize genotype evaluated Blue Nile in season 2008 at Damazin

\begin{tabular}{|c|c|c|c|c|c|c|c|c|c|}
\hline Genotype & $\begin{array}{c}\text { Days to } 50 \% \\
\text { Tasseling }\end{array}$ & $\begin{array}{c}\text { Stem } \\
\text { diameter } \\
(\mathrm{Cm})\end{array}$ & $\begin{array}{c}\text { No. of } \\
\text { leavel } \\
\text { plant }\end{array}$ & $\begin{array}{c}\text { leaf area } \\
(\mathrm{Cm})\end{array}$ & $\begin{array}{c}\text { Cob } \\
\text { Barnes }\end{array}$ & $\begin{array}{c}\text { Cob } \\
\text { diameter } \\
(\mathrm{cm})\end{array}$ & $\begin{array}{c}\text { Cob } \\
\text { weight } \\
(\mathrm{cm})\end{array}$ & $\begin{array}{c}\text { yield } \\
\text { /plant } \\
\text { (g) }\end{array}$ & yield $t / h$ \\
\hline M45 & 57.4 & 2.0 & 12.9 & 358.7 & 1.9 & 4.2 & 116.1 & 98.4 & 6.1 \\
\hline H1 & 59.0 & 1.9 & 12.2 & 342.5 & 1.7 & 3.8 & 112.7 & 106.0 & 5.5 \\
\hline B1 & 56.7 & 2.3 & 13.6 & 375.1 & 1.5 & 3.8 & 120.0 & 84.8 & 6.7 \\
\hline B2 & 61.2 & 2.2 & 14.9 & 301.1 & 1.4 & 4.6 & 107.8 & 84.3 & 6.5 \\
\hline B3 & 56.6 & 2.4 & 13.2 & 272.9 & 1.9 & 3.5 & 95.6 & 99.0 & 5.7 \\
\hline B4 & 58.2 & 2.3 & 13.1 & 257.9 & 1.4 & 3.6 & 125.1 & 86.5 & 4.2 \\
\hline B5 & 57.4 & 2.5 & 12.5 & 292.1 & 1.7 & 4.5 & 113.9 & 81.2 & 5.4 \\
\hline D1 & 57.2 & 2.3 & 13.4 & 422.2 & 1.6 & 4.1 & 118.0 & 76.6 & 4.0 \\
\hline D2 & 59.0 & 2.7 & 14.9 & 356.4 & 1.7 & 4.1 & 119.8 & 83.3 & 4.4 \\
\hline D3 & 57.6 & 1.8 & 13.6 & 320.2 & 1.7 & 4.3 & 112.4 & 85.2 & 4.3 \\
\hline D4 & 55.3 & 1.8 & 12.6 & 386.8 & 2.4 & 3.7 & 94.3 & 81.2 & 4.7 \\
\hline D5 & 55.2 & 2.3 & 12.7 & 355.0 & 2.2 & 4.2 & 98.0 & 77.3 & 5.2 \\
\hline D6 & 57.8 & 2.0 & 11.7 & 408.6 & 1.7 & 3.6 & 95.1 & 76.7 & 5.3 \\
\hline $\mathrm{R} 1$ & 56.2 & 1.9 & 14.6 & 231.2 & 1.5 & 3.8 & 87.9 & 75.3 & 6.3 \\
\hline $\mathrm{R} 2$ & 57.5 & 1.8 & 11.7 & 324.1 & 2.2 & 3.9 & 85.2 & 78.9 & 4.1 \\
\hline R3 & 56.4 & 1.8 & 12.6 & 192.4 & 1.6 & 4.0 & 118.8 & 85.8 & 4.1 \\
\hline $\mathrm{R} 4$ & 60.1 & 1.8 & 13.9 & 362.0 & 2.4 & 3.6 & 111.2 & 88.9 & 4.2 \\
\hline R5 & 58.6 & 2.2 & 14.7 & 336.0 & 2.5 & 3.7 & 100.4 & 83.9 & 4.0 \\
\hline M45XB1 & 59.7 & 3.0 & 12.1 & 303.8 & 2.7 & 4.1 & 93.4 & 83.4 & 3.9 \\
\hline M45XB2 & 56.1 & 2.1 & 14.5 & 453.2 & 1.5 & 3.9 & 95.8 & 81.2 & 3.4 \\
\hline M45XB3 & 58.3 & 2.5 & 13.8 & 349.3 & 2.7 & 4.3 & 118.9 & 74.0 & 3.4 \\
\hline M45XB4 & 58.0 & 2.0 & 13.3 & 289.6 & 3.1 & 3.9 & 105.7 & 83.3 & 3.4 \\
\hline M45XB5 & 56.8 & 1.7 & 15.3 & 382.3 & 1.8 & 3.8 & 102.4 & 82.9 & 3.7 \\
\hline M45XD1 & 58.3 & 2.2 & 11.4 & 354.6 & 1.9 & 4.4 & 96.0 & 81.7 & 4.1 \\
\hline M45XD2 & 57.4 & 2.1 & 12.4 & 229.2 & 1.6 & 4.4 & 100.0 & 82.4 & 4.5 \\
\hline M45XD3 & 60.9 & 2.0 & 13.6 & 326.9 & 1.9 & 3.9 & 103.6 & 80.9 & 5.3 \\
\hline M45XD4 & 55.9 & 2.2 & 11.2 & 233.1 & 2.9 & 3.4 & 99.6 & 79.0 & 4.2 \\
\hline M45XD5 & 59.5 & 2.1 & 13.4 & 265.6 & 2.1 & 4.0 & 105.3 & 79.8 & 3.3 \\
\hline M45XD6 & 58.3 & 1.8 & 11.8 & 346.7 & 3.1 & 3.9 & 88.1 & 77.1 & 3.8 \\
\hline M45XR1 & 57.1 & 1.9 & 11.2 & 344.8 & 2.6 & 4.5 & 95.9 & 80.0 & 4.1 \\
\hline M45XR2 & 57.5 & 2.3 & 14.6 & 298.9 & 2.1 & 3.8 & 95.9 & 82.3 & 4.9 \\
\hline M45XR3 & 55.4 & 2.8 & 13.9 & 240.9 & 1.9 & 4.0 & 94.9 & 74.7 & 5.0 \\
\hline M45XR4 & 56.0 & 2.1 & 12.1 & 331.6 & 2.5 & 4.2 & 93.9 & 81.3 & 3.5 \\
\hline M45XR5 & 57.7 & 2.5 & 14.3 & 387.1 & 3.6 & 3.9 & 116.1 & 83.0 & 3.1 \\
\hline H1XB1 & 57.1 & 2.3 & 13.7 & 372.9 & 2.6 & 3.1 & 106.1 & 84.1 & 3.2 \\
\hline H1XB2 & 59.8 & 2.4 & 13.6 & 347.8 & 1.7 & 3.7 & 108.4 & 84.9 & 2.4 \\
\hline
\end{tabular}




\begin{tabular}{|l|l|l|l|l|l|l|l|l|l|}
\hline H1XB3 & 60.1 & 2.4 & 12.0 & 361.0 & 3.9 & 3.5 & 87.2 & 56.6 & 1.8 \\
\hline H1XB4 & 58.3 & 2.0 & 13.0 & 276.0 & 2.9 & 3.6 & 75.6 & 76.5 & 2.0 \\
\hline H1XB5 & 60.0 & 2.4 & 11.3 & 270.9 & 1.9 & 2.9 & 87.1 & 75.9 & 2.0 \\
\hline H1XD1 & 58.6 & 2.1 & 11.8 & 208.4 & 1.4 & 3.4 & 84.7 & 81.5 & 2.1 \\
\hline H1XD2 & 57.4 & 2.4 & 14.3 & 268.9 & 1.6 & 4.0 & 86.3 & 82.1 & 2.4 \\
\hline H1XD3 & 58.1 & 2.1 & 13.9 & 302.1 & 1.5 & 3.8 & 105.9 & 84.0 & 3.2 \\
\hline H1XD4 & 56.2 & 2.4 & 14.9 & 293.6 & 2.4 & 3.3 & 113.4 & 82.5 & 3.1 \\
\hline H1XD5 & 60.0 & 2.7 & 12.1 & 276.5 & 2.2 & 4.0 & 94.5 & 79.6 & 3.2 \\
\hline H1XD6 & 57.1 & 2.8 & 11.9 & 282.3 & 2.8 & 3.7 & 85.8 & 84.2 & 3.4 \\
\hline H1XR1 & 56.7 & 1.9 & 12.8 & 272.5 & 2.7 & 4.1 & 69.2 & 83.2 & 2.6 \\
\hline H1XR2 & 60.4 & 2.0 & 14.1 & 283.2 & 1.6 & 3.7 & 75.0 & 77.6 & 2.1 \\
\hline H1XR3 & 54.8 & 1.9 & 13.8 & 312.8 & 2.3 & 3.4 & 82.0 & 83.4 & 2.3 \\
\hline H1XR4 & 56.5 & 2.3 & 12.6 & 274.6 & 2.1 & 4.0 & 100.8 & 79.7 & 3.3 \\
\hline H1XR5 & 57.0 & 2.4 & 12.8 & 299.3 & 2.8 & 3.9 & 106.3 & 84.5 & 2.9 \\
\hline $\begin{array}{l}\text { Overall } \\
\text { means }\end{array}$ & 57.8 & 2.2 & 13.1 & 314.7 & 2.1 & 3.9 & 100.1 & 82.0 & 3.9 \\
\hline C.V\% & 4.25 & 13.01 & 7.99 & 12.64 & 17.85 & 9.12 & 4.59 & 3.51 & 12.48 \\
\hline
\end{tabular}

\subsection{Cob Bareness\%}

In the two seasons at location, significant differences in percent of cob bareness percentage were detected among the different genotype, which showed highly significant differences for the crosses in both seasons and location, significant for line and tester in both seasons and location, while highly significant differences were detected for genotype X tester, in both seasons and location ( Table 3). The combined analysis of variance (table 7) showed highly significant differences for seasons, genotype and seasons $\mathrm{X}$ genotype in both seasons and locations as shown in Table (4). The higher percentage of cob bareness were observed by $\mathrm{H}_{1}$ $\mathrm{X} \mathrm{B}_{3} \quad(3.9 \%)$ and the lowest one were recorded by $\mathrm{H}_{1} \mathrm{X} \mathrm{D}_{\mathrm{I}}$ $(1.4 \%)$ in first season, while in the second season the highest one were given by genotype $\mathrm{H}_{1} \mathrm{X} \mathrm{B}_{3} \quad(3.9 \%)$ and the lowest one were given by genotype $\mathrm{H}_{1} \mathrm{X} \mathrm{D}_{3}(1.4 \%)$.The overall mean for this character was $2.1 \%$ in both seasons, and the coefficient of variation of was 17.985 in both seasons.

\subsection{Cob Diameter (Cm)}

Table (2) indicated that Individual analysis of variance were significant for cob diameter among the evaluated genotype in first season while it was highly significant in second season. The mean squares for genotype $\mathrm{X}$ tester analysis showed highly significant differences for crosses in first season and location while highly significant for line and the taster were detected in second season and location (Table 3 ). On the other hand highly significant differences for genotype $\mathrm{X}$ tester in both seasons and location were recorded. The combined analysis of variance showed highly significant differences for seasons, genotypes, and genotype $\mathrm{X}$ seasons in location as revealed in Table (4). The cob diameter ranged from $2.9 \mathrm{Cm}$ for genotype $\mathrm{H}_{1} \mathrm{X} \mathrm{B}_{5}$ to 4.6 $\mathrm{Cm}$ for genotype $\mathrm{B}_{2}$ in both seasons. The overall mean of cob diameter of $3.5 \mathrm{Cm}$ in both seasons, and the coefficient of variation was $9.12 \%$ in both seasons.

\subsection{Cob Weight (gm)}

The individual analysis of variance for cob weight indicated significant differences among the evaluated genotypes at location and in both seasons (Table 2). The mean squares for genotype $\mathrm{X}$ tester analysis (table 6) showed highly significant differences for crosses at location and season as shown in Table (3). Non significant differences were detected for genotype in both seasons and location, while the tester showed significant differences in first season. The combined analysis of variance showed no significant for seasons, highly significant for genotypes and seasons $\mathrm{X}$ genotypes (Table 4). On the other hand, non- significant differences were recorded for season in location. In the first season the cob weight ranged from $75 \mathrm{~g}$ for $\mathrm{H}_{1} \mathrm{X} \mathrm{R}_{2}$ to $120 \mathrm{~g}$ for $B_{1}$. The heaviest genotype was $B_{1}(120.0 \mathrm{~g})$, but the lightest one was $\mathrm{H}_{1} \times \mathrm{R}_{2}(75.0 \mathrm{~g})$, while in the second season the heaviest cob weight ranged from $75 \mathrm{~g}$ for $\mathrm{H}_{1} \mathrm{X} \mathrm{R}_{2}$ to $125.1 \mathrm{~g}$ for--- $\mathrm{B}_{4}$---- and the lightest one was $H_{1} \times$ $R_{2}(75.0 \mathrm{~g})$ with the overall mean of cob weight as $1000.1 \mathrm{~g}$ in the first season and $101.2 \mathrm{~g}$ in the second season with the coefficient of variation as $4.59 \%$ in both seasons.

\subsection{Yield per Plant (G)}

The individual analysis of variance for yield per plant 
indicated significant differences among the evaluated genotypes in location and season (Table 2). The genotype X tester analysis of variance showed highly significant differences for crosses in both season and location, while non- significant were recorded for genotypes and tester in both seasons and location. On the other hand, highly significant differences were recorded for genotype $\mathrm{X}$ tester in both seasons and location (Table 3). The combined analysis of variance showed highly significant differences for seasons, genotypes, season $\mathrm{X}$ genotype (Table 4). The yield/plant ranged from $56.5 \mathrm{~g}$ for genotype $\mathrm{H}_{1} \mathrm{X} \mathrm{B}_{3}$ to highest $106 \mathrm{~g}$ for genotype. $\mathrm{H}_{1}$ in both seasons, with an overall mean yield/plant as $82 \mathrm{~g}$ in both seasons and the coefficient of variation was $3.51 \%$ in both seasons.

\subsection{Grain Yield (t/ha)}

The individual analysis of variance showed significant differences among the evaluated genotypes for this trait at location and seasons (Table 2). The genotype $\mathrm{X}$ tester analysis of variance showed highly significant differences for crosses in both season and location, while nonsignificant differences for genotypes in both seasons and location. On the other hand, the testers showed highly significant differences in second season (Table 3). Combined analysis of variance showed highly significant differences for seasons, genotypes. X season in both seasons as shown in Table (4). In the first season, the yield ranged from $2.0 \mathrm{t} / \mathrm{ha}$ for $\mathrm{R}_{1}$ to $6.7 \mathrm{t} / \mathrm{ha}$ for $\mathrm{H}_{1} \mathrm{X} \mathrm{D}_{4}$, while during the second season, the yield ranged from $1.8 \mathrm{t} / \mathrm{ha}$ for $\mathrm{H}_{1} \mathrm{X} \mathrm{B}_{3}$ to $6.7 \mathrm{t} / \mathrm{ha}$ for $\mathrm{M}_{45} \mathrm{X} \mathrm{D}_{3}$, with the overall mean --- yield of 4.1 and $3.9 \mathrm{t} / \mathrm{ha}$ in the first season and second seasons, respectively. The coefficient of variation was $9.987 \%$ in the first season and $12.48 \%$ in second one.

\section{Conclusion}

a- a wide range of phenotypic variability was detected among the evaluated genotypes at the end of the two seasons for most of the triats studied. This variability can be exploited in the improvement of this crop.

$b$ - The significance $(p>0.05)$ of years, seasons, and the interactions of years and seasons, years with genotypes, seasons with genotypes and years with genotypes indicated the importance of genotypes X environment interaction. Nevertheless, evaluation over more seasons and years is essential.

c- A great amount of variability as measured by the range of means and the co-efficient of variations was expressed by the different characters which were also illustrated.

d- In combining ability analysis mean squares due to lines were larger than that due to testers for most of the characters indicating that variation in general in combining ability effects was mainly due to variation among lines.

e- Portioning of mean square of crosses to lines and lines to testers interactions revealed significant differences for line $\mathrm{X}$ testers for all characters indicating the existence of variation among the crosses.

\section{REFERENCES}

[1] Banziger M., Edmeades GO., and Lafitte HR. 1999b. Selection for drought tolerance increases maize yield across a range of nitrogen levels. Grop. Sci. 39. 1035-1040.

[2] Edmeades GO., Bolanos J., Banziger M., Chapman SG., Ortega A., Lafitte HR., Fischer KS., and Pandex S. 1997c. Recurrent selection under managed drought stress improve grain yields in tropical maize in Edmeades GO., Baziger M., Mickelson HR., and Pena-Valdiva CB.(Eds), Developing drought and Loon- Tolerant Maize. Proceedings of a symposium, March 25-29, 1996, CIMMYT, EL Batan Mexico, D.E., CIMMYT, PP.415 - 425.

[3] FAO. 2003. Bulletin of Statistics, Vol.4.No.1. Food and Agriculture Organization of the United Nations. Rome, 2003.

[4] Gomez KA., and Gomez AA. 1984. Statistical procedures for Agriculture Research. ${ }^{3}$ th Edition Johan Wiley. New York.

[5] Heisey PW. , and Edmeades GO. 1999. Maize production in drought stressed environments; Technical options and research resource allocation. In CIMMYT.

[6] Koutsika - Sotiriou M. 1999 Hybrid seed production in maize In Bas a, A.s. (Ed). Heterosis and Hybrid seed production in Agronomic Crops. Food products Press, New York, pp. $25-64$.

[7] Pingali PL. and Pandey S. 2000. Meeting world Maize Needs; Technological opportunities and priorities for public sector. In CIMMYT, 1999/2000. World Maize Fast and Trends, part 1. CIMMYT, Mexico City, pp.1-3.

[8] Vasal SK., Cordova H., Beck DL., and Edmeades GO. 1997. Choices among breeding procedures and strategic for developing stress tolerant maize germplasm. In Edmeades GO., Banziger M., Mickelson HR., and Pena- Valdiva CB. (Eds.). Developing drought and loon Tolerant Maize proceedings of a symposium, March 25-29, 1996, CIMMYT. EL Ban, Mexico. D. F., CIMMYT, PP.336-347. 